\title{
Real-Time Three-Phase Line Loss Calculation for Distribution Network Based on Electric Characteristic Measuring Terminal
}

\author{
Jian $\mathrm{Li}^{1}$, Chenxi Wang ${ }^{2}$, Yuancan Zhuang ${ }^{2}$ and Jiangwen $\mathrm{Yi}^{2}$ \\ ${ }^{1}$ Electric Power Research Institute of Guangdong Power Grid Corporation, Guang Zhou, 510080, China \\ ${ }^{2}$ School of Electric Power Engineering, South China University of Technology, 381 Wushan Road, Guangzhou, China
}

\begin{abstract}
Line loss rate is an important economic index of distribution network. In the paper, a new real-time three-phase line loss calculation method based on characteristic measurement is proposed for calculating the line loss of distribution network. The state characteristics of distribution network is measured by the new smart metering terminal, such that the change state of the electric load can be described in a simple way, relieving the pressure in transmitting and processing the large amount of real-time measurement data, while the calculating accuracy of the line loss of distribution network is ensured. The loop current method is used to calculate the three-phase line loss of distribution network, respectively, depending not on the topology and parameters of the distribution network. The effect of three-phase unbalance and distributed generation problems also can be considered effectively. The proposed method is applied to a practical distribution network, the results show its feasibility and effectiveness fully.
\end{abstract}

Keywords-distribution network; real-time line loss calculation; characteristic measurement; loop current method; three-phase unbalance; distributed generation

\section{INTRODUCTION}

Line loss rate is not only an important economic index to evaluate the operation state of power systems, but also a reference to its planning, operation and technological innovation. Since the distribution network in China had not been paid enough attention in the past years, its line loss rate is comparing higher than the transmission network [1].

Nowadays, much research has been done on the calculation of theoretical line loss of distribution network. The existing methods include the root-mean-square current method, average current method, maximum current method, equivalence resistance method and so on [2-3]. These methods just need a small amount of measurements, which are easy to be obtained via the coulomb meter, thus they are widely used in the practical engineering. However, these methods are proposed based on some idealized assumptions ignoring the difference of voltages, phase angles and load change shapes of distribution network. Thus, the results calculated by these methods have large errors in most cases. It is found in some practical applications that the calculation errors of these methods are even up to $-23 \% \sim 29 \%$ [4].

In order to improve the accuracy, the line loss is also calculated based on the power flow methods, among which the forward and backward substitution method and Newton-Raphson method are the most concerned ones. The forward and backward substitution method with good convergence is suitable to calculate the line loss of radial distribution network. However, it is difficult in dealing with the distribution network with looped structure [5]. The Newton-Raphson method performs well in calculating the line loss of distribution network with looped structure, but its convergence is sensitive to the initial value [6-7]. Due to the long-range radiation of distribution network, the true value always deviates from the initial value largely, making the Newton-Raphson method be not convergent [8].

In order to solve these problems, the power flow methods are improved from different aspects [9]. However, these improved methods are dependent on the fixed network structure and accurate line parameters. In the practical operation process, the structure of distribution network is changed some times. The line parameters also can't be measured accurately for the complicated factors such as the changing of climate [10].

In the paper, a new real-time three-phase line loss calculation method is proposed. The characteristic measuring technology combined with the loop current method is used to calculate the three-phase line loss of distribution network, respectively. The effect of three-phase unbalance, changing network structure and distributed generation integration all can be considered effectively by the proposed method.

\section{LiNE LOSS CALCULATION BASED ON LOOP CURRENT METHOD}

\section{A. Line Loss Calculation of Distribution Network}

According to loop current method, a loop can be seen as a path from the first end feeder to a terminal branch, and the power loss of this loop can be calculated by multiplying its voltage with current.

As shown in Figure I, where the distribution network has $N$ branches, it can form $N$ loops with the common first end feeder and each terminal branch, respectively. The branch current can be regarded as the loop current, and the voltage difference between two ends can be regarded as the loop voltage. Then the power loss can be calculated as

$$
\Delta P_{i, m}=U_{s, m} I_{i, m} \cos \left(\alpha_{s, m}-\theta_{i, m}\right)-U_{i, m} I_{i, m} \cos \left(\alpha_{i, m}-\theta_{i, m}\right)
$$




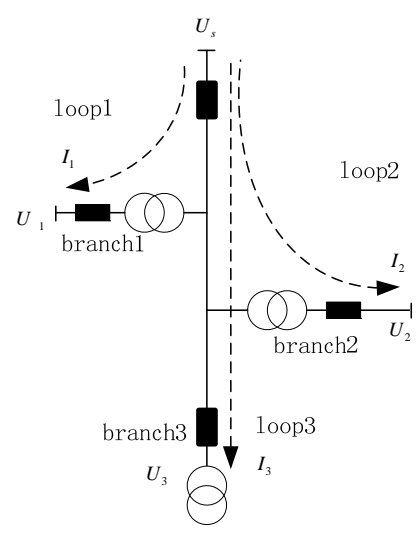

FIGURE I. BASIC PRINCIPLE OF LINE LOSS BASED ON LOOP CURRENT METHOD

where $\Delta P_{i, m}$ is power loss of loop $i ; U_{s, m}$ and $\alpha_{s, m}$ are the $m$ phase voltage amplitude and angle of the first end feeder, respectively; $U_{i, m}$ and $\alpha_{i, m}$ are the $m$ phase voltage amplitude and angle of branch $i$, respectively; $I_{i, m}$ and $\theta_{i, m}$ are the $m$ phase current amplitude and angle of branch $i$, respectively.

The line loss $\Delta W_{i, m}$ in period $T$ can be calculated as

$$
\Delta W_{i, m}=\int_{0}^{T}\left[U_{s, m} I_{i, m} \cos \left(\alpha_{s, m}-\theta_{i, m}\right)-U_{i, m} I_{i, m} \cos \left(\alpha_{i, m}-\theta_{i, m}\right)\right] d t
$$

In order to measure the characteristics conveniently, the voltage and current phasor are changed into the algebraic form. Then the line loss $\Delta W_{i, m}$ in period $T$ can be calculated as

$$
\Delta W_{i, m}=\int_{0}^{T}\left(U_{s, m}^{p} I_{i, m}^{p}+U_{s, m}^{q} I_{i, m}^{q}-U_{i, m}^{p} I_{i, m}^{p}-U_{i, m}^{q} I_{i, m}^{q}\right) d t
$$

where $U_{s, m}^{p}$ and $U_{s, m}^{q}$ are the real part and image part of $m$ phase voltage of the first end feeder, respectively; $U_{i, m}^{p}$ and $U_{i, m}^{q}$ are the real part and image part of $m$ phase voltage of branch $i$, respectively; $I_{i, m}^{p}, I_{i, m}^{q}$ are the real part and image part of $m$ phase current of branch $i$, respectively.

According to the principle of superposition, the total line loss is equal to the sum of each loop line loss. Thus the total line loss $\Delta W$ can be calculated as

$$
\Delta W=\sum_{i=1}^{N} \sum_{m=1}^{3} \Delta W_{i, m}
$$

where $\Delta W$ is the total line loss of distribution network.

In the above method, the three-phase unbalance problem can be solved effectively as the line loss of each phase is calculated, respectively. The topological structure and parameter values are also omitted in the calculation process. Therefore, it is suitable to be applied in the distribution network where the topological structure changes some times and its true parameter values are unknown.

B. Line Loss Calculation Including Distributed Generation

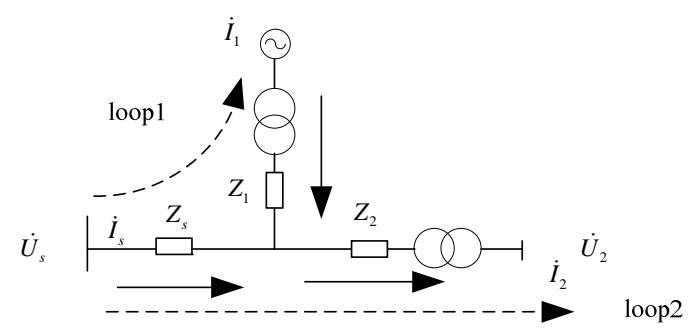

FIGURE II. BASIC PRINCIPLE OF LINE LOSS OF DISTRIBUTION NETWORK INCLUDING DISTRIBUTED GENERATION

The proposed method is also suitable to calculate the line loss including the distributed generation. Take Figure II as example, the distributed generation and load are connected in loop 1 and loop 2, respectively. The positive direction of current is defined as the direction from the first end feeder to each branch. According to Kirchhoff's law, the current phasor can be calculated as

$$
\dot{I}_{s}=\dot{I}_{1}+\dot{I}_{2}
$$

where $\dot{I}_{s}, \dot{I}_{1}$ and $\dot{I}_{2}$ are current phasors of the first end feeder, branch 1 and branch 2, respectively.

The total line loss $W$ in period $T$ can be calculated as

$$
W^{\prime}=\int_{0}^{T}\left(I_{1}^{2} R_{1}+I_{2}^{2} R_{2}+I_{s}^{2} R_{s}\right) d t
$$

where $R_{s}, R_{1}$ and $R_{2}$ are the resistances of the first end feeder, branch 1 and branch 2 , respectively.

According to (3), the total loss can be calculated as

$$
\begin{aligned}
W= & \int_{0}^{T} \sum_{i=1}^{2}\left(U_{s}^{p} I_{i}^{p}+U_{s}^{q} I_{i}^{q}-U_{i}^{p} I_{i}^{p}-U_{i}^{q} I_{i}^{q}\right) d t \\
= & \int_{0}^{T} \sum_{i=1}^{2}\left[\left(U_{s}^{p}-U_{i}^{p}\right) I_{i}^{p}+\left(U_{s}^{q}-U_{i}^{q}\right) I_{i}^{q}\right] d t \\
= & \int_{0}^{T} \sum_{i=1}^{2}\left[\left(I_{s}^{p} R_{s}-I_{s}^{q} X_{s}+I_{i}^{p} R_{i}-I_{i}^{q} X_{i}\right) I_{i}^{p}+\right. \\
& \left.\left(I_{s}^{p} X_{s}+I_{s}^{q} R_{s}+I_{i}^{p} X_{i}+I_{i}^{q} R_{i}\right) I_{i}^{q}\right] d t \\
= & \int_{0}^{T}\left(I_{1}^{2} R_{1}+I_{2}^{2} R_{2}+I_{s}^{2} R_{s}-I_{s}^{p} I_{s}^{q} X_{S}+I_{s}^{q} I_{s}^{p} X_{S}\right) d t \\
= & \int_{0}^{T}\left(I_{1}^{2} R_{1}+I_{2}^{2} R_{2}+I_{s}^{2} R_{s}\right) d t
\end{aligned}
$$

It shows that the line loss calculation result based on the loop current method is as the same as (6). Thus, the loop 
current method is also suitable to the line loss calculation of including distributed generation.

\section{LINE LOSS CHARACTERISTIC MEASUREMENT}

\section{A. Line Loss Characteristics}

In (3), the line loss can be calculated in discrete form as

$$
\Delta W_{i, m}=\sum_{k=1}^{M}\left(U_{s, m, k}^{p} I_{i, m, k}^{p}+U_{s, m, k}^{q} I_{i, m, k}^{q}-U_{i, m, k}^{p} I_{i, m, k}^{p}-U_{i, m, k}^{q} I_{i, m, k}^{q}\right) \Delta T_{k}(8)
$$

where $U_{s, m, k}^{p}$ and $U_{s, m, k}^{q}$ are the real part and image part of $m$ phase voltage of the first end feeder in period $k ; U_{i, m, k}^{p}$ and $U_{i, m, k}^{q}$ are the real part and image part of $m$ phase voltage of branch $i$ in period $k ; I_{i, m, k}^{p}, I_{i, m, k}^{q}$ are the real part and image part of $m$ phase current of branch $i$ in period $k ; \Delta T_{k}$ is the time interval in period $k$; $M$ is the total number of periods.

Equation (8) shows that the shorter the time interval is, the higher the calculation accuracy will be. But considering the communication limit of the distribution network, it is impossible to transmit the measurement in a very short interval. A more feasible approach is to measure the line loss characteristics, which represent the line loss fully, in an appropriate interval, and then transmit them into the master station for the accurate line loss calculation.

In the root-mean-square current method, the line loss is calculated by the mean square root of current in one period. It can be proved that the mean square root of current in a period is equal to the effect of actual current when the line loss is calculated. In this sense, the root mean square of current is a good characteristic for line loss calculation. But the traditional root mean square method can only process the effective value of current other than that in the algebraic form. Thus, it is necessary to propose the new line loss characteristics based on the root-mean-square current method.

For each branch of distribution network, a smart metering terminal can be set to measure the line loss characteristics. First, translate the phase current into the algebraic form as

$$
\begin{gathered}
I_{i, m, k}^{p}=I_{i, m, k} \cos \left(\theta_{i, m, k}\right) \\
I_{i, m, k}^{q}=I_{i, m, k} \sin \left(\theta_{i, m, k}\right)
\end{gathered}
$$

Then the current characteristics can be calculated as

$$
\begin{gathered}
\tilde{I}_{i, m, k}^{p}=\sqrt{\frac{\int I_{i, m, k}^{p}{ }^{2} d t}{T}} \\
\tilde{I}_{i, m, k}^{q}=\sqrt{\frac{\int I_{i, m, k}^{q}{ }^{2} d t}{T}}
\end{gathered}
$$

where $\tilde{I}_{i, m, k}^{p}$ and $\tilde{I}_{i, m, k}^{q}$ are the real part and image part of $m$ phase current of branch $i$ in period $k$, respectively.

Similarly, the voltage characteristics can be calculated as

$$
\begin{gathered}
U_{i, m, k}^{p}=U_{i, m, k} \cos \left(\theta_{i, m, k}\right) \\
U_{i, m, k}^{q}=U_{i, m, k} \sin \left(\theta_{i, m, k}\right) \\
\tilde{U}_{i, m, k}^{p}=\sqrt{\frac{\int U_{i, m, k}^{p} d t}{T}} \\
\tilde{U}_{i, m, k}^{q}=\sqrt{\frac{\int U_{i, m, k}^{q} d t}{T}}
\end{gathered}
$$

where $\tilde{U}_{i, m, k}^{p}$ and $\tilde{U}_{i, m, k}^{q}$ are real part and image part of the $m$ phase voltage of branch $i$ in period $k$, respectively.

When the line loss characteristics in (11), (12), (15) and (16) are obtained, they can be substituted into (8) for the calculation of line loss.

\section{B. Phase Change of Characteristics in Both Sides of Transformer}

The transformers are generally classified into the special and public ones. For the special transformer, whose loss is taken charged by the users, its loss should not be taken into calculation. Since the measuring terminals of special transformer are generally installed in the high-voltage side, the characteristics can be used directly for the calculation of line loss. However, for the public transformer whose measuring terminals are installed in low-voltage side, its characteristics measured in the low-voltage side have to be translated into the high-voltage side for the calculation of line loss in $10 \mathrm{kV}$.

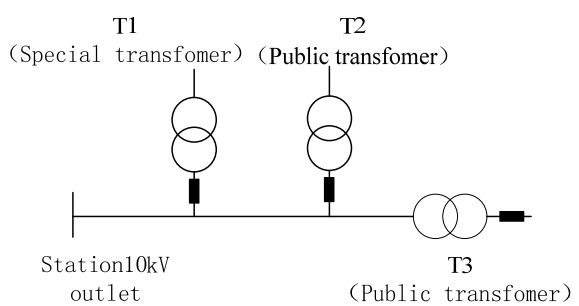

FIGURE III. INSTALLATION LOCATION OF METERING TERMINALS OF DIFFERENT TYPES OF TRANSFORMERS

Considering the three-phase unbalance problem of distribution network, the symmetrical component method is applied to decompose the characteristics in low-voltage side into different sequence components as

$$
\left[\begin{array}{c}
\tilde{U}_{i, a, k}^{1} \\
\tilde{U}_{i, a, k}^{2}
\end{array}\right]=\frac{1}{3}\left[\begin{array}{ccc}
1 & \alpha & \alpha^{2} \\
1 & \alpha^{2} & \alpha
\end{array}\right]\left[\begin{array}{c}
\tilde{U}_{i, a, k} \\
\tilde{U}_{i, b, k} \\
\tilde{U}_{i, c, k}
\end{array}\right]
$$




$$
\left[\begin{array}{c}
\tilde{I}_{i, a, k}^{1} \\
\tilde{I}_{i, a, k}^{2}
\end{array}\right]=\frac{1}{3}\left[\begin{array}{lll}
1 & \alpha & \alpha^{2} \\
1 & \alpha^{2} & \alpha
\end{array}\right]\left[\begin{array}{c}
\tilde{I}_{i, a, k} \\
\tilde{I}_{i, b, k} \\
\tilde{I}_{i, c, k}
\end{array}\right]
$$

where $\alpha=e^{j 120^{\circ}} ; \tilde{U}_{i, a, k}^{1}$ and $\tilde{U}_{i, a, k}^{2}$ are the positive-sequence and negative-sequence component of A phase voltage characteristics, respectively; $\tilde{I}_{i, a, k}^{1}$ and $\tilde{I}_{i, a, k}^{2}$ are the positive-sequence component and negative-sequence component of A phase current characteristics, respectively; $\tilde{U}_{i, a, k}, \tilde{U}_{i, b, k}$ and $\tilde{U}_{i, c, k}$ are the A, B and C phase voltage characteristics of the public transformer in the low-voltage side, respectively; $\tilde{I}_{i, a, k}, \tilde{I}_{i, b, k}$ and $\tilde{I}_{i, c, k}$ are the A, B and C three-phase current characteristics of public transformer in the low-voltage side, respectively.

In general, the public transformers possess the $\mathrm{D} / \mathrm{Y}_{0}-11$ connection structure. According to the transformer ratio and phase variation of each sequence current, the characteristics in the high-voltage side can be calculated as

$$
\begin{aligned}
& {\left[\begin{array}{c}
\tilde{U}_{i, A, k} \\
\tilde{U}_{i, B, k} \\
\tilde{U}_{i, C, k}
\end{array}\right]=K\left[\begin{array}{ll}
\tilde{U}_{i, a, k}^{1} & \tilde{U}_{i, a, k}^{2} \\
\alpha^{2} \tilde{U}_{i, a, k}^{1} & \alpha \tilde{U}_{i, a, k}^{2} \\
\alpha \tilde{U}_{i, a, k}^{1} & \alpha^{2} \tilde{U}_{i, a, k}^{2}
\end{array}\right]\left[\begin{array}{c}
e^{j 30^{\circ}} \\
e^{-j 30^{\circ}}
\end{array}\right]} \\
& {\left[\begin{array}{c}
\tilde{I}_{i, A, k} \\
\tilde{I}_{i, B, k} \\
\tilde{I}_{i, C, k}
\end{array}\right]=K\left[\begin{array}{ll}
\tilde{I}_{i, a, k}^{1} & \tilde{I}_{i, a, k}^{2} \\
\alpha^{2} \tilde{I}_{i, a, k}^{1} & \alpha \tilde{I}_{i, a, k}^{2} \\
\alpha \tilde{I}_{i, a, k}^{1} & \alpha^{2} \tilde{I}_{i, a, k}^{2}
\end{array}\right]\left[\begin{array}{c}
e^{j 30^{\circ}} \\
e^{-j 30^{\circ}}
\end{array}\right]}
\end{aligned}
$$

where $\tilde{U}_{i, a, k}, \tilde{U}_{i, b, k}$ and $\tilde{U}_{i, c, k}$ are the A, B and C phase voltage characteristics of the public transformer high-voltage side, respectively; $\tilde{I}_{i, a, k}, \tilde{I}_{i, b, k}$ and $\tilde{I}_{i, c, k}$ are the $\mathrm{A}, \mathrm{B}$ and $\mathrm{C}$ phase current characteristics of the public transformer in high-voltage side, respectively.

\section{TEST ANALYSIS}

\section{A. Test Analysis of Pilot 1}

The proposed method is applied to a real distribution network in Dongguan, Guangdong, whose network structure is shown in Figure IV. The equivalent resistance method, which is the major line loss calculation method in China Southem Power Grid Company Limited, is also used for the comparing its effectiveness with the proposed method. When the proposed method is applied, the time interval is set to be 15 minutes. The calculation results are shown in Figure 4 and Tab. I-III.

Figure $\mathrm{V}$ shows the real-time line loss of pilot 1 in a typical day. It can be seen that the line loss increases gradually from 7 o'clock, and then stay in a large value from 9 to 17 o'clock. The reason is that the loads in pilot 1 are mainly the small processing factories which consume mainly the electric power during the day time, thus the line loss of this pilot at these times is relatively large.
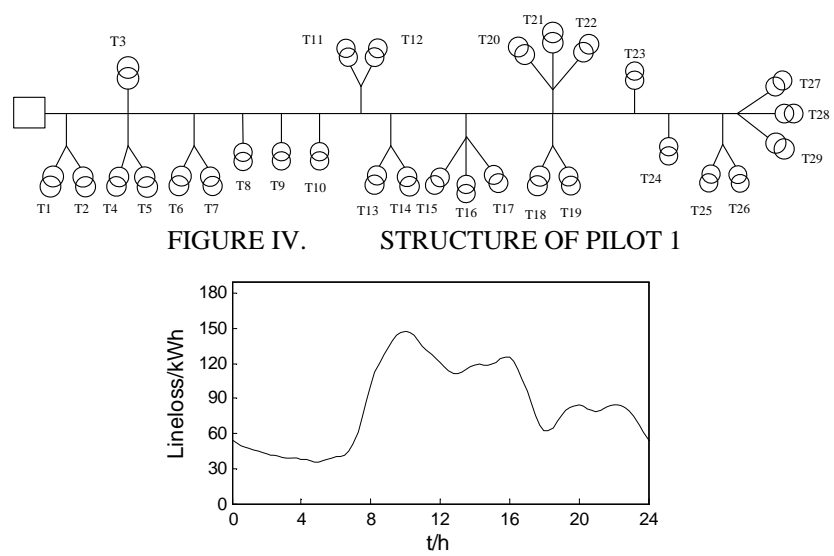

FIGURE V. CALCULATION RESULTS OF REAL-TIME LINE LOSS DURING THE TYPICAL DAY IN PILOT 1

TABLE I. CALCULATION RESULT OF LINE LOSS OF EACH LOOP IN PILOT 1

\begin{tabular}{|c|c|c|c|c|c|}
\hline Loop & Loss & Loop & Loss & Loop & Loss \\
\hline loop1 & 241.1708 & loop11 & 562.6290 & loop21 & 13.7974 \\
\hline loop2 & 155.4010 & loop12 & 24.7449 & loop22 & 4.9946 \\
\hline loop3 & 37.5871 & loop13 & 101.1405 & loop23 & 25.1765 \\
\hline loop4 & 61.8677 & loop14 & 22.9689 & loop24 & 26.4538 \\
\hline loop5 & 72.3295 & loop15 & 3.73189 & loop25 & 55.054 \\
\hline loop6 & 156.7959 & loop16 & 50.2675 & loop26 & 30.1814 \\
\hline loop7 & 38.4249 & loop17 & 15.7338 & loop27 & 16.3542 \\
\hline loop8 & 139.3663 & loop18 & 49.9786 & loop28 & 10.8543 \\
\hline loop9 & 71.0575 & loop19 & 5.0040 & loop29 & 18.2546 \\
\hline 1oop10 & 77.2643 & loop20 & 2.0538 & & \\
\hline
\end{tabular}

\section{(unit:KWH)}

As shown in Table I-III, calculation results of the line loss provided by various methods are relatively small. The reason is that the power loss of the large number of special transformers is not included in the calculation of line loss of distribution network. When the calculation results of various methods are compared, it can be seen that the calculation results of the proposed method and the statistical line loss are close. Their relative deviation is only $5.45 \%$. However, the relative deviation between the equivalent resistance method and the statistical line loss is $65.3 \%$. This certifies the effectiveness of the proposed method in some extent.

TABLE II. CALCULATION RESULTS OF THE PROPOSED METHOD IN PILOT 1

\begin{tabular}{|c|c|c|c|c|}
\hline Project & Total loss & A phase & B phase & C phase \\
\hline $\begin{array}{c}\text { line loss } \\
\text { value }\end{array}$ & 2110.6377 & 684.5334 & 760.4336 & 665.6687 \\
\hline
\end{tabular}

TABLE III. CALCULATION RESULTS OF DIFFERENT METHODS IN PILOT 1

\begin{tabular}{|c|c|c|c|}
\hline Method & $\begin{array}{c}\text { The method of } \\
\text { equivalent } \\
\text { resistance }\end{array}$ & $\begin{array}{c}\text { Statistical line } \\
\text { loss }\end{array}$ & $\begin{array}{c}\text { The proposed } \\
\text { method }\end{array}$ \\
\hline $\begin{array}{c}\text { line loss } \\
\text { rate }\end{array}$ & $1.69 \%$ & $4.87 \%$ & $4.6044 \%$ \\
\hline
\end{tabular}




\section{B. Test Analysis of Pilot 2}

The proposed method is used in another pilot including the distribution generations in Dongguan. Its structure is shown in the Figure VII, where T18, T19 and T20 are connected to the distributed generation. Select a typical day, and calculate the line loss by the proposed method and the equivalent resistance method, respectively. The real-time line loss is shown in Figure 7. The line loss value of each loop, each phase and the total line loss are shown in Table IV - V. The results provided by various methods are shown in the Table VI.

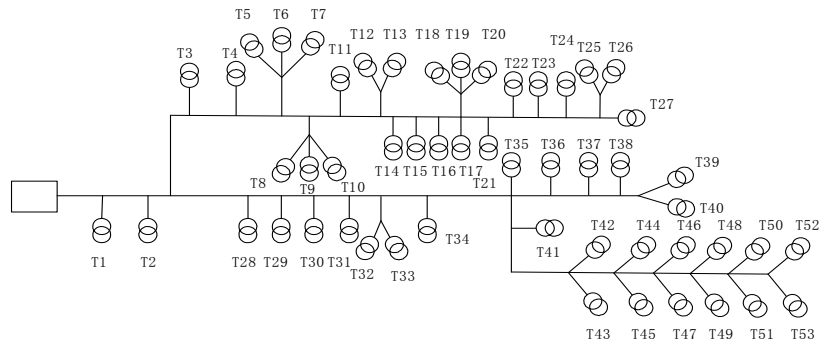

FIGURE VI. STRUCTURE OF PILOT 1

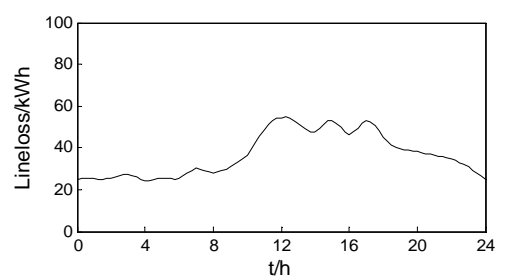

FIGURE VII. CALCULATION RESULTS OF REAL TIME LINE LOSS DURING THE TYPICAL DAY IN PILOT 2

It can be found that, the relative deviation between the equivalent resistance method and the statistical line loss is $25.46 \%$, while relative deviation between the proposed method and the statistical line loss is $7.53 \%$. Thus the proposed method is more suitable to calculate the line loss of distribution network including the distributed generations.

TABLE IV. CALCULATION RESULT OF LINE LOSS OF EACH LOOP IN PILOT 2

\begin{tabular}{|c|c|c|c|c|c|}
\hline Loop & Loss & Loop & Loss & Loop & Loss \\
\hline loop1 & 47.6621 & loop19 & -30.7173 & loop37 & 32.6563 \\
\hline loop2 & 4.3117 & loop20 & -5.3944 & loop38 & 20.1001 \\
\hline loop3 & 13.3312 & loop21 & 7.4261 & loop39 & 10.3580 \\
\hline loop4 & 28.1072 & loop22 & 64.7861 & loop40 & 25.6212 \\
\hline loop5 & 13.1453 & loop23 & 76.8067 & loop41 & 9.0686 \\
\hline loop6 & 9.3116 & loop24 & 62.5157 & loop42 & 11.0932 \\
\hline loop7 & 3.6143 & loop25 & 44.2165 & loop43 & 24.0056 \\
\hline loop8 & 16.6288 & loop26 & 8.4729 & loop44 & 8.7373 \\
\hline loop9 & 12.3217 & loop27 & 6.6838 & loop45 & 30.0599 \\
\hline 1oop10 & 85.2622 & loop28 & 4.2814 & loop46 & 12.5702 \\
\hline loop11 & 86.1164 & loop29 & 2.7841 & loop47 & 13.5282 \\
\hline loop12 & 7.4437 & loop30 & 5.6842 & loop48 & 72.3427 \\
\hline loop13 & 4.7193 & loop31 & 72.5294 & loop49 & 15.9137 \\
\hline loop14 & 24.3202 & loop32 & 3.9007 & loop50 & 21.2246 \\
\hline loop15 & 12.7484 & loop33 & 28.3387 & loop51 & 29.3955 \\
\hline loop16 & 8.4928 & loop34 & 31.6356 & loop52 & 22.1839 \\
\hline loop17 & 5.4343 & loop35 & 25.2015 & loop53 & 13.2262 \\
\hline loop18 & -14.3579 & loop36 & 32.4061 & & \\
\hline
\end{tabular}

(UNIT:kWh)
TABLE V. CALCULATION RESULTS OF THE PROPOSED METHOD IN PILOT 2

\begin{tabular}{|c|c|c|c|c|}
\hline Project & Total loss & A phase & B phase & C phase \\
\hline loss & 2110.6337 & 684.5354 & 760.4336 & 665.6687 \\
\hline
\end{tabular}

(UNIT:kWh)

TABLE VI. CALCULATION RESULTS OF DIFFERENT METHODS IN PILOT 2

\begin{tabular}{|c|c|c|c|}
\hline Method & $\begin{array}{c}\text { The method of } \\
\text { equivalent } \\
\text { resistance }\end{array}$ & $\begin{array}{c}\text { Statistical line } \\
\text { loss }\end{array}$ & $\begin{array}{c}\text { The method of } \\
\text { loop cureent }\end{array}$ \\
\hline $\begin{array}{c}\text { line loss } \\
\text { rate }\end{array}$ & $1.69 \%$ & $4.87 \%$ & $4.6044 \%$ \\
\hline
\end{tabular}

(UNIT:kWh)

\section{CONCLUSIONS}

A real-time three-phase line loss calculation method for distribution network based on characteristic measurement is proposed in this paper. Then it is applied in the real distribution network in Dongguan, Guangdong. The main conclusions are as follows:

(1)The proposed method is able to calculate the three-phase real-time line loss of distribution network accurately when the three phases are unbalance;

(2)The proposed method can describe the impact of distributed generations on the line loss fully, and calculate the line loss of distribution network accurately not matter the distributed generations are included or not;

(3)The proposed method can be applied conveniently, depending not on the topology and parameters of the distribution network.

\section{ACKNOWLEDGEMENTS}

Fund Project: China southern power grid science and technology projects(GDKJ00000049)

\section{REFERENCES}

[1] Z. G. Yu, X. Y. Chen, and H. Liu, Power loss power network. BeiJing: China Power Press, 2002.

[2] L. X. Yang, H. Liu, and H. Y. Zhang, "Improved load shape coefficient method in low voltage line loss calculation," Power Demand Side Management. Nanjing, vol. 11, pp. 29-32, August 2009.

[3] H. H. Chen, and Q. C Cheng, "Theory and practice of distribution network loss calculation through method of equivalent resistance," Guangdong Electric Power. Guangzhou, vol.17, pp. 5-8, March 2004.

[4] K. Wang, and L. P. Liu, "Error Analysis on $10 \mathrm{kV}$ Line Loss with Theoretical Calculating Method,” Journal of Electric Power. Taiyuan, vol.17, pp. 251-253, 256. August 2002.

[5] Y. J. Hu, J. M. Zhang, and D. Y. Liu, "Calculation of multi-source distribution network loss based on back/ forward sweep method and measurement resource,” Electric Power Automation Equipment Nanjing, vol. 29, pp. 55-59, December 2009.

[6] T. l. Liu, S. Wang, and Z. Zhang, "Newton- Raphson method for theoretical line loss calculation of low-voltage distribution transformer district by using the load electrical energy,” Power System Protection and Control. Xuchang, vol. 43, pp. 143-148, October 2015.

[7] Q. Y. Sun, H. M. Chen, and J. N. Yang, "Analysis on convergence of Newton-like power flow algorithm,” Proceedings of the CSEE. Beijing, vol. 34, pp. 2196 -2200, July 2014.

[8] Q. S Xu, Z. Z. Liu, Y. B. Yang, "Improved method of distribution network three-phase power flow calculation," Proceedings of the CSU-EPSA. Tianjing, vol. 29, pp. 23-29, September 2014.

[9] X. Y. Yang, J. D. Duan, and W. Y. Yang, "Power flow calculation based 
on power losses sensitivity for distribution system with distributed generation,” Power System Technology. Beijing, vol. 33, pp. 39-143, September 2009.

[10] Y. H. Xiao, and K. F. Gao, “Analysis and application of $10 \mathrm{kV}$ distribution network loop closing," Electro technical Application. Beijing, vol. 34, pp. 18-23, June 2015. 